\title{
Prediction of cycle life of flexible pipe bellows
}

\author{
Abdulrahman Th. Mohammad ${ }^{1}$, Jasim Abdulateef ${ }^{2}$, Zaid Hammoudi ${ }^{2}$ \\ ${ }^{1}$ Baqubah Technical Institute, Middle Technical University, Baghdad, Iraq \\ ${ }^{2}$ Mechanical Engineering Department, Diyala University, Diyala, Iraq
}

Email address:

abd20091976@gmail.com (A. Th. Mohammad),jmabdulateef@gmail.com (J. Abdulateef), zshaaa@yahoo.com (Z. Hammoudi)

\section{To cite this article:}

Abdulrahman Th. Mohammad, Jasim Abdulateef, Zaid Hammoudi. Prediction of Cycle Life of Flexible Pipe Bellows. International Journal of Mechanical Engineering and Applications. Vol. 3, No. 1, 2015, pp. 6-15. doi: 10.11648/j.ijmea.20150301.12

\begin{abstract}
This paper aims to investigate the relationship between maximum stresses produced and cycle life of different shaped bellows expansion joints. Flexible pipe bellows which have been selected in the present study are made of stainless (STS 304) and (STS 316) material based on two types of (axial, and axial with control ring) with three shapes of bellow section (U-shape, $\Omega$-shape and disc-shape). Our calculation is done using simulation model written by MATLAB. The simulation results show that, U-shaped bellow has smaller internal pressure-induced stress, longer fatigue life, and is more suited for higher internal pressure situations. The most essential bellow design factor is the correct specification of the bellow movement requirements.
\end{abstract}

Keywords: Cycle Life of Bellows, Flexible Pipe, Bellows Movement

\section{Introduction}

Bellows are widely used as the element of expansion joint in various piping systems. Piping systems for industrial plants often suffer excessive deformations or displacements caused by heat expansion, vibration, non uniform subsidence of ground, etc. [1]. Bellows function is to absorb regular or irregular expansion and contraction in such piping systems.

A metal bellows consist of individual convolutions which have a uniform inside and outside diameters, constant pitch or spacing, and the parallel planes of each convolution. The flexibility of the convolutions gives the bellows the ability to absorb axial, angular and lateral displacement individually or in combination [2]. Axial motion is extension or compression of the bellows along the longitudinal centerline ( $x$-axis). The motion is absorbed equally by the convolutions.

The bellows cycle life or bellows fatigue life is defined as the total number of complete cycles which can be expected from the expansion joint based on data tabulated from tests performed at room temperature. A bellows cycle can also be defined as one complete movement of an expansion joint from initial position to the extreme position and return to initial position. The bellows cycle life is affected by the following design factors [3].

- Operating pressure

- Operating temperature

- Bellows Material
- Thickness of bellows and number of plies/layers

- The movement per convolution

- Pitch, depth and shape of convolutions

- Heat treatment of bellows

Besides, the physical limitations of deflecting a bellows without damaging it, the design is normally based on specified cycles movements for a given fatigue life at the operating pressure and temperature.

The correct specification of bellows movement requirements is one of the most essential factors in the successful application of this product. The axial, lateral and angular movements must be realistically stated along with the corresponding cycle life. One of the most common mistakes made is to overstate these values in an attempt to obtain a conservative design [2].

EJMA [4] defined fatigue life analysis in terms of meridional stresses and have mentioned that the other type of stresses, if signification, can also be caused a fatigue failure.

A number of studies have been done to predict the fatigue failure in bellows expansion joints [5-7].

Tingxin et al. [8], studied experimentally the toroid-shaped bellows behavior. The results showed that, compared with U-shaped bellows, toroidal bellows have longer fatigue life, smaller internal pressure-induced stress, stronger ability to resist internal pressure instability.

Zhu et al. [9], studied experimentally the effect of environmental medium on corrosion fatigue life for U-shaped 
bellows expansion joints. The results showed that the presence of corrosive medium accelerates both crack initiation and propagation rates and reduces the failure life for the expansion joints.

Pierce and Evans [10], analyzed the failure of a metal bellows flexible hose which subject to multiple pressure cycles. It was found that by cycling the pressure on the hose and applying a pressure much greater than the yield capabilities of the hose, this combination of yielding and cycling, caused a burst failure.

The metal bellows presents an ongoing challenge: the prediction of an accurate cycle life. Bellows manufacturers require a method for detection of bellows cycle life to failure. The objective of the present work is to study the relationship between maximum stresses produced in the bellows and their cycle life. The flexible pipe bellows used are made of (STS 304) and (STS 316) material based on two types of (axial, and axial with control ring). The solution process involves three shapes of bellow section (U-shape, $\Omega$-shape and disc-shape).

\section{Cycle Life of Flexible Pipe Bellows}

When bellows deflect, the motion is absorbed by bending of the sidewalls of each convolution. The associated stress caused by this motion is the deflection stress or EJMA stress [11]. This stress runs longitudinal to the bellows centerline. The maximum value of deflection stress is located in the sidewall of each convolution near the crest or root.

Expansion joints are designed to operate with a value for deflection stress that far exceeds the yield strength of the bellows material. This means that most expansion joints will take a permanent set at the rated axial, angular or lateral motion. Expansion joint bellows are rarely designed to operate in the elastic stress range [11]. Therefore the bellows will eventually fatigue after a finite number of movement cycles. It is important to specify a realistic cycle life as a design consideration when ordering an expansion joint. An overly conservative cycle life requirement can result in a bellows design that is so long and soft that it is subject to squirm failure

\section{Mathematical Model}

A bellows is a flexible seal and convoluted portion of an expansion joint which is designed to flex when thermal movements occur in the piping system. The number of convolutions depends upon the amount of movement the bellows must accommodate or the force that must be used to accomplish this deflection. The convoluted element must be strong enough circumferentially to withstand the internal pressure of the system. The longitudinal load (pressure thrust) must then be absorbed by some other type of device [11].

The internal pressure exerts an axial force equal to the pressure times the internal cross-section area of the pipe and also induces stresses in the circumferential direction. It can be assumed that the displacement $(e) \mathrm{mm}$ per one bellow corrugation gives a stress due to deflection produced in the bellows, $\left(\sigma_{e}\right) \mathrm{kg} / \mathrm{mm}^{2}$, and the internal pressure or the external pressure can also be added to these stresses is $\left(\sigma_{p}\right) \mathrm{kg} / \mathrm{mm}^{2}$. The maximum stress $\left(\sigma_{m}\right)$ produced for three shapes of bellow section (U-shape, $\Omega$-shape and disc-shape) are approximately expressed as follows $[12,13]$ :

$$
\sigma_{m}=\sigma_{e}+\sigma_{p}
$$

For U-shape corrugation

$$
\sigma_{m_{1}}=\frac{1.5 * E^{*} t^{*} e}{2 * \sqrt{W} * H^{1.5}}+\frac{P * H^{2}}{2 * t^{2} * m}
$$

For $\Omega$-shape corrugation

$$
\sigma_{m_{2}}=\frac{1.5 * E * t * e}{2 * H^{2}}+\frac{P^{*} H^{2}}{t^{*} m}
$$

For disc-type corrugation

$$
\sigma_{m_{3}}=\frac{3 * E^{*} t^{*} e}{2 * H^{2}}+\frac{P^{*} H^{2}}{2 * t^{2}}
$$

Bellows meridional bending stress due to internal pressure $\left(\mathrm{kg} / \mathrm{mm}^{2}\right)$ is written as[13]:

$$
\sigma_{P}=\frac{P^{*} H^{2}}{2 * t^{2} * m}
$$

where $\sigma_{P} \leq \sigma_{y}$

Also, the bellows circumferential membrane stress due to internal pressure $\left(\mathrm{kg} / \mathrm{mm}^{2}\right)$ is written as [13]:

$$
\sigma_{e}=\frac{P^{*} D_{P}^{*} W}{t^{*} m^{*}(1.142 * W+2 * H)}
$$

where $\sigma_{e} \leq \sigma_{a}$

Finally, the relationship between maximum stresses $\left(\sigma_{m}\right)$ produced in the bellows and its life cycle $(N)$ is expressed as [12]:

$$
\begin{aligned}
& N_{A}=\left(\frac{563}{\sigma_{m}}\right)^{3.5} \\
& N_{B}=\left(\frac{1125}{\sigma_{m}}\right)^{3.5}
\end{aligned}
$$

\section{Calculation Procedure}

Figure (1) shows the proposed bellow design that used in our calculation. The materials of bellows that used are both of (STS 304) and (STS 316). The solution procedure is done by solving the above equations using simulation model written by MATLAB [14]. The design parameters of bellows are presented in Table (1). 


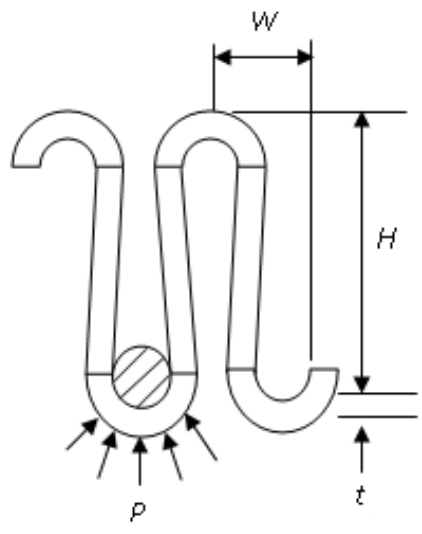

Figure 1. Bellow design

\section{Results and Discussion}

It is important to predict a realistic cycle life as a design consideration when ordering bellows expansion joints. Thus, a life cycle prediction program by MATLAB is done to investigate the relationship between maximum stresses produced in the bellows and the predicted cycle life. The model depends on the data that mentioned in Table (1) as input parameters under different operating temperature [13].

Tables ( 2 and 3 ) show that the maximum total stresses acts on the flexible pipe bellows is limited by the internal and external pressure or circumference tensile stress. When the design temperature increases, the value of $\left(\sigma_{y}\right)$ decreases. As a result, the pressure and $\sigma_{m}$ decrease while both of allowable and broken cycle lives increase.

Table 1. Design parameters of bellow

\begin{tabular}{lcc}
\hline PARAMETERS & VALUE & UNIT \\
\hline Height of bellow corrugation, $(H)$ & 60 & $\mathrm{~mm}$ \\
Half pitch of bellow corrugation, $(W)$ & 30 & $\mathrm{~mm}$ \\
Thickness of bellow, $(t)$ & 1.5 & $\mathrm{~mm}$ \\
Effective diameter of bellow, $\left(D_{P}\right)$ & 346 & $\mathrm{~mm}$ \\
Allowable tensile stress, $\left(\sigma_{a}\right)$ & 10.1 & $\mathrm{Kg} / \mathrm{mm}^{2}$ \\
Yield strength, $\left(\sigma_{y}\right)$ & 16.1 & $\mathrm{Kg} / \mathrm{mm}^{2}$ \\
Number of plies, $(m)$ & 1 & ---- \\
\hline
\end{tabular}

Table 2. The maximum total stresses applied on the flexible pipe bellows of both (axial, and axial with control ring) type, (STS 304)

\begin{tabular}{|c|c|c|c|c|c|c|c|}
\hline \multicolumn{8}{|l|}{ Axial type } \\
\hline \multirow{4}{*}{ U-shape } & $T\left(\mathrm{C}^{0}\right)$ & 100 & 200 & 300 & 400 & 500 & 600 \\
\hline & $\sigma_{m}\left(\mathrm{~kg} / \mathrm{mm}^{2}\right)$ & 35.71 & 33.35 & 31.65 & 24.91 & 29.81 & 23 \\
\hline & $N_{A}$ & 15560 & 19767 & 23739 & 54887 & 29276 & 72235 \\
\hline & $N_{B}$ & 175496 & 222945 & 267747 & 619050 & 330192 & 814713 \\
\hline \multirow{3}{*}{$\Omega$ - shape } & $\sigma_{m}\left(\mathrm{~kg} / \mathrm{mm}^{2}\right)$ & 64.49 & 59.293 & 56 & 38.62 & 53.25 & 36.15 \\
\hline & $N_{A}$ & 1965 & 2637 & 3209 & 11823 & 3842 & 14898 \\
\hline & $N_{B}$ & 22172 & 29752 & 36203 & 33350 & 43342 & 167987 \\
\hline \multirow[t]{2}{*}{ Disc-shape } & $N_{A}$ & 8268 & 10329 & 12380 & 25527 & 15229 & 32006 \\
\hline & $N_{B}$ & 93251 & 116496 & 139628 & 287908 & 171761 & 360982 \\
\hline \multicolumn{8}{|c|}{ Axial type with control ring } \\
\hline \multirow{3}{*}{ U-shape } & $\sigma_{m}\left(\mathrm{~kg} / \mathrm{mm}^{2}\right)$ & 99.47 & 90.79 & 86.25 & 84.5 & 81.73 & 83.73 \\
\hline & $N_{A}$ & 431 & 593 & 710 & 763 & 857 & 803 \\
\hline & $N_{B}$ & 4865 & 6697 & 8014 & 8607 & 9676 & 9064 \\
\hline \multirow{2}{*}{$\Omega$ - shape } & $\sigma_{m}\left(\mathrm{~kg} / \mathrm{mm}^{2}\right)$ & 382.87 & 346.39 & 331.46 & 323.74 & 312.68 & 322.86 \\
\hline & $N_{B}$ & 43 & 61 & 72 & 78 & 88 & 79 \\
\hline \multirow{3}{*}{ Disc- shape } & $\sigma_{\mathrm{m}}\left(\mathrm{kg} / \mathrm{mm}^{2}\right)$ & 106.55 & 97.55 & 93.33 & 90.68 & 87.85 & 88.93 \\
\hline & $N_{A}$ & 339 & 461 & 539 & 596 & 666 & 638 \\
\hline & $N_{B}$ & 3824 & 5208 & 6080 & 6725 & 7515 & 7200 \\
\hline
\end{tabular}

Table 3. The maximum total stresses applied on the flexible pipe bellows of both (axial, and axial with control ring) type, (STS 316)

\begin{tabular}{|c|c|c|c|c|c|c|c|}
\hline Axial type & & & & & & & \\
\hline \multirow{4}{*}{ U-shape } & $T\left(\mathrm{C}^{0}\right)$ & 100 & 200 & 300 & 400 & 500 & 600 \\
\hline & $\sigma_{m}\left(\mathrm{~kg} / \mathrm{mm}^{2}\right)$ & 36.27 & 34.79 & 33.65 & 32.5 & 31 & 26.47 \\
\hline & $N_{A}$ & 14735 & 17048 & 19459 & 21613 & 25499 & 44375 \\
\hline & $N_{B}$ & 166194 & 192284 & 216065 & 243766 & 287592 & 500489 \\
\hline \multirow{3}{*}{$\Omega$ - shape } & $\sigma_{m}\left(\mathrm{~kg} / \mathrm{mm}^{2}\right)$ & 66 & 63.34 & 61.66 & 60 & 55.28 & 45.8 \\
\hline & $N_{A}$ & 1806 & 2093 & 2300 & 2530 & 3371 & 6512 \\
\hline & $N_{B}$ & 20371 & 23609 & 25943 & 28543 & 38022 & 73452 \\
\hline \multirow[t]{2}{*}{ Disc-shape } & $N_{A}$ & 7900 & 9130 & 10351 & 11850 & 13575 & 21636 \\
\hline & $N_{B}$ & 89100 & 102973 & 116744 & 133651 & 153106 & 244023 \\
\hline
\end{tabular}


Table 3. (continued)

\begin{tabular}{llcccccc}
\hline \multicolumn{2}{l}{ Axial type with control ring } & & & & & \\
\multirow{5}{*}{ U-shape } & $\sigma_{\mathrm{m}}\left(\mathrm{kg} / \mathrm{mm}^{2}\right)$ & 104.27 & 98 & 94.85 & 93.3 & 87.3 & 70.4 \\
& $N_{A}$ & 365 & 454 & 509 & 539 & 680 & 1441 \\
\multirow{5}{*}{ - shape } & $N_{B}$ & 4125 & 5127 & 5746 & 6085 & 7672 & 16256 \\
& $\sigma_{m}\left(\mathrm{~kg} / \mathrm{mm}^{2}\right)$ & 404.47 & 378.79 & 367.46 & 363.34 & 337.88 & 265.2 \\
& $N_{A}$ & 3 & 4 & 4 & 5 & 6 & 14 \\
Disc-shape & $N_{B}$ & 35 & 45 & 50 & 52 & 67 & 157 \\
& $\sigma_{m}\left(\mathrm{~kg} / \mathrm{mm}^{2}\right)$ & 11.35 & 104.78 & 101.33 & 99.48 & 93.36 & 76.1 \\
& $N_{A}$ & 296 & 359 & 404 & 431 & 538 & 1099 \\
& $N_{B}$ & 3278 & 4055 & 4559 & 4863 & 6073 & 12404 \\
\hline
\end{tabular}

Figures (2 and 3) show the effect of total stress on the allowable and broken cycle life for the three shapes of bellows (STS 304) with both of axial and axial with control ring types respectively. As shown in Figures, both the $N_{A}$ and $N_{B}$ in axial type are increased with decreasing the total stress. It can be seen inFig.2, when the total stress changes from 30 to 60
$\mathrm{Kg} / \mathrm{mm}^{2}$, the $N_{A}$ and $N_{B}$ reach to the maximum value of about $90 \times 10^{3}$ and $800 \times 10^{3}$ respectively in case of U-shape corrugation. In axial type with control ring, the $N_{A}$ and $N_{B}$ reach to the maximum value of about $1 \times 10^{3}$ and $10 \times 10^{3}$ respectively in case of U-shape bellows as shown in Fig.3.

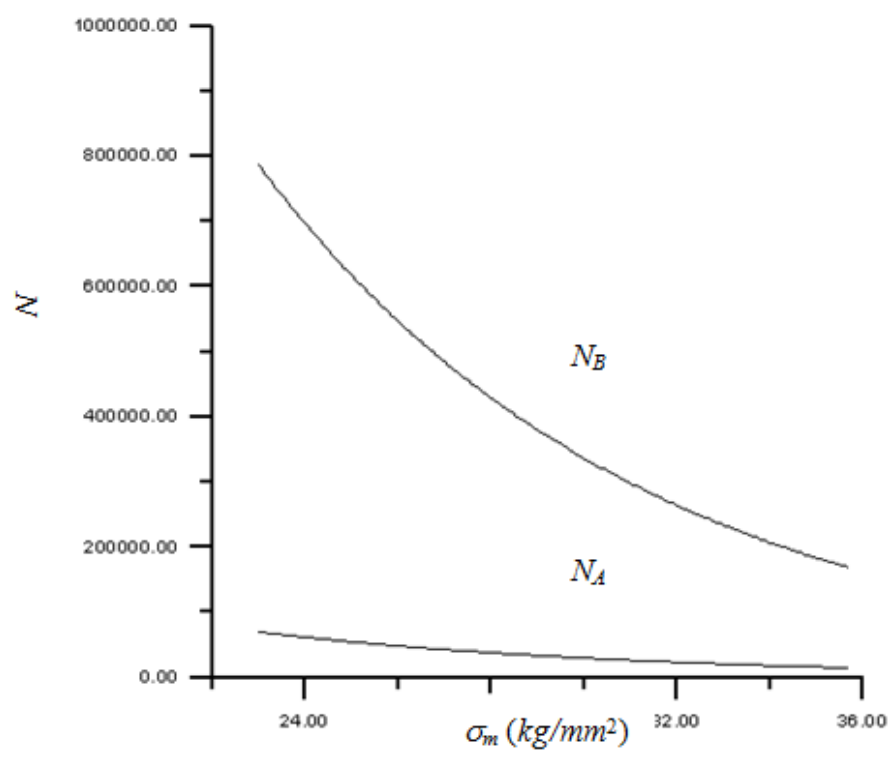

(a) U-shape bellow

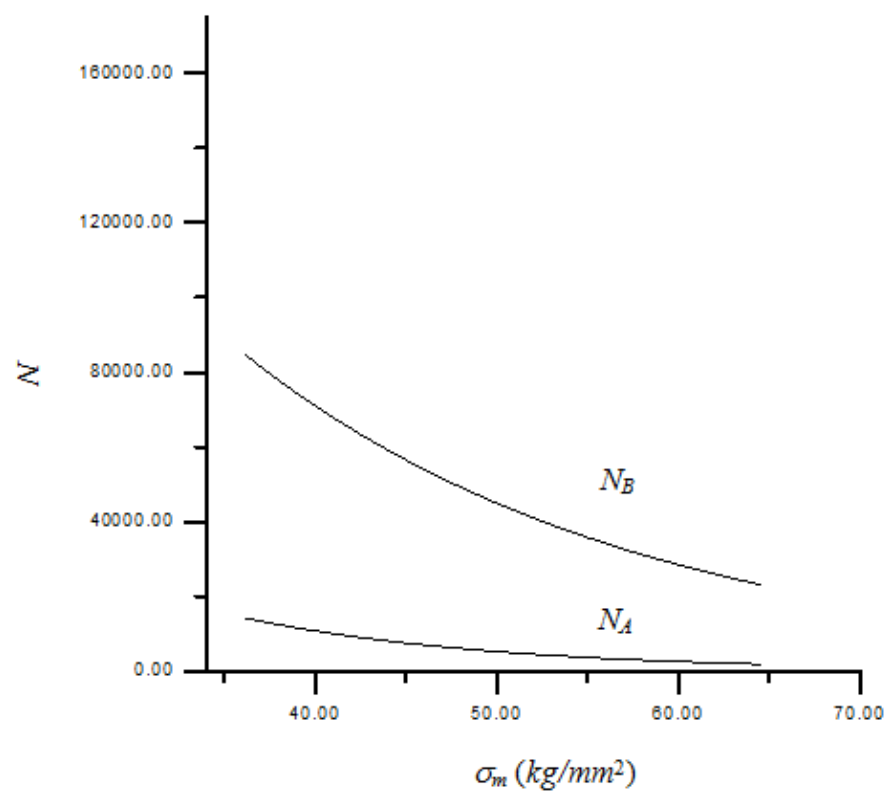

(b) $\Omega$-shape bellow 


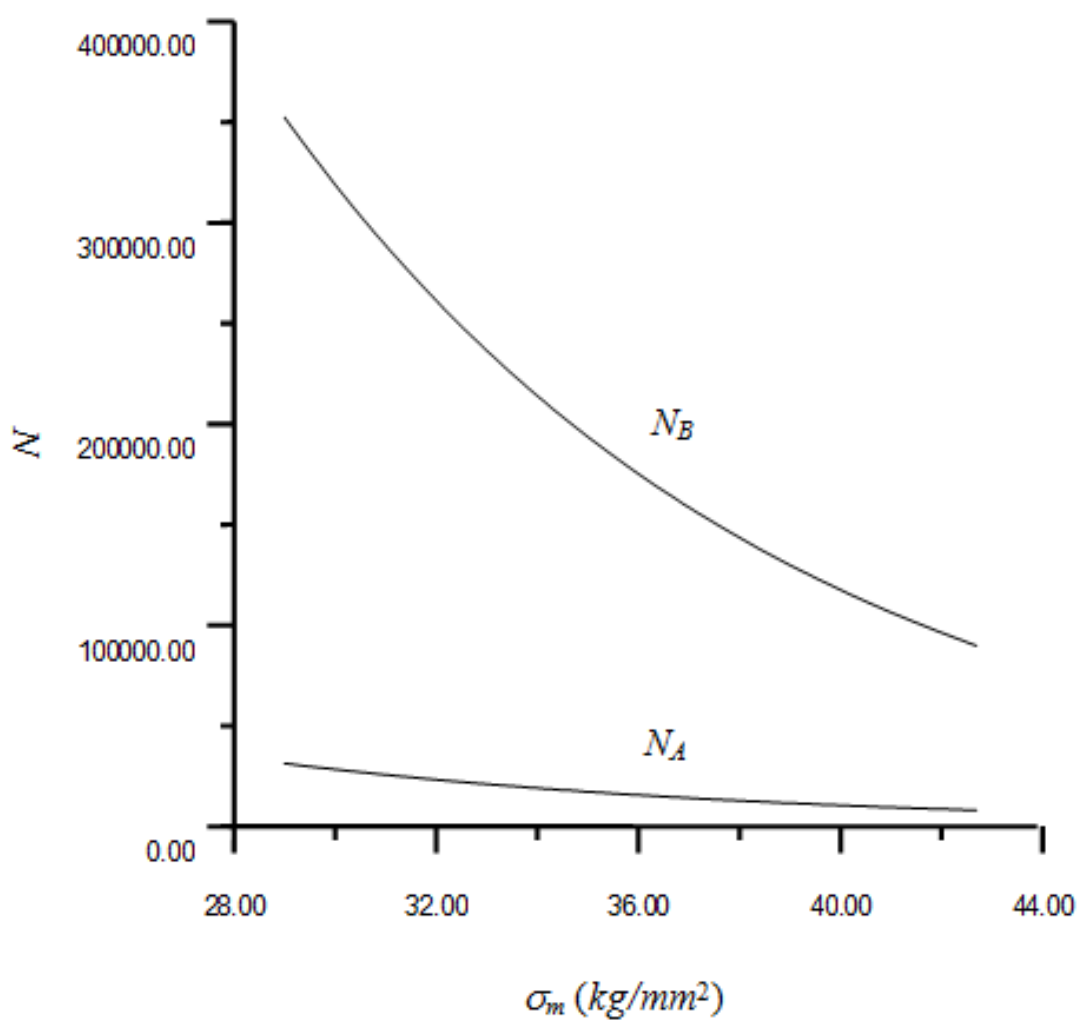

(c) disc-shape bellow

Figure 2. The relationship between ( $\sigma_{m}$ and $N$ ) for axial type, (STS 304)

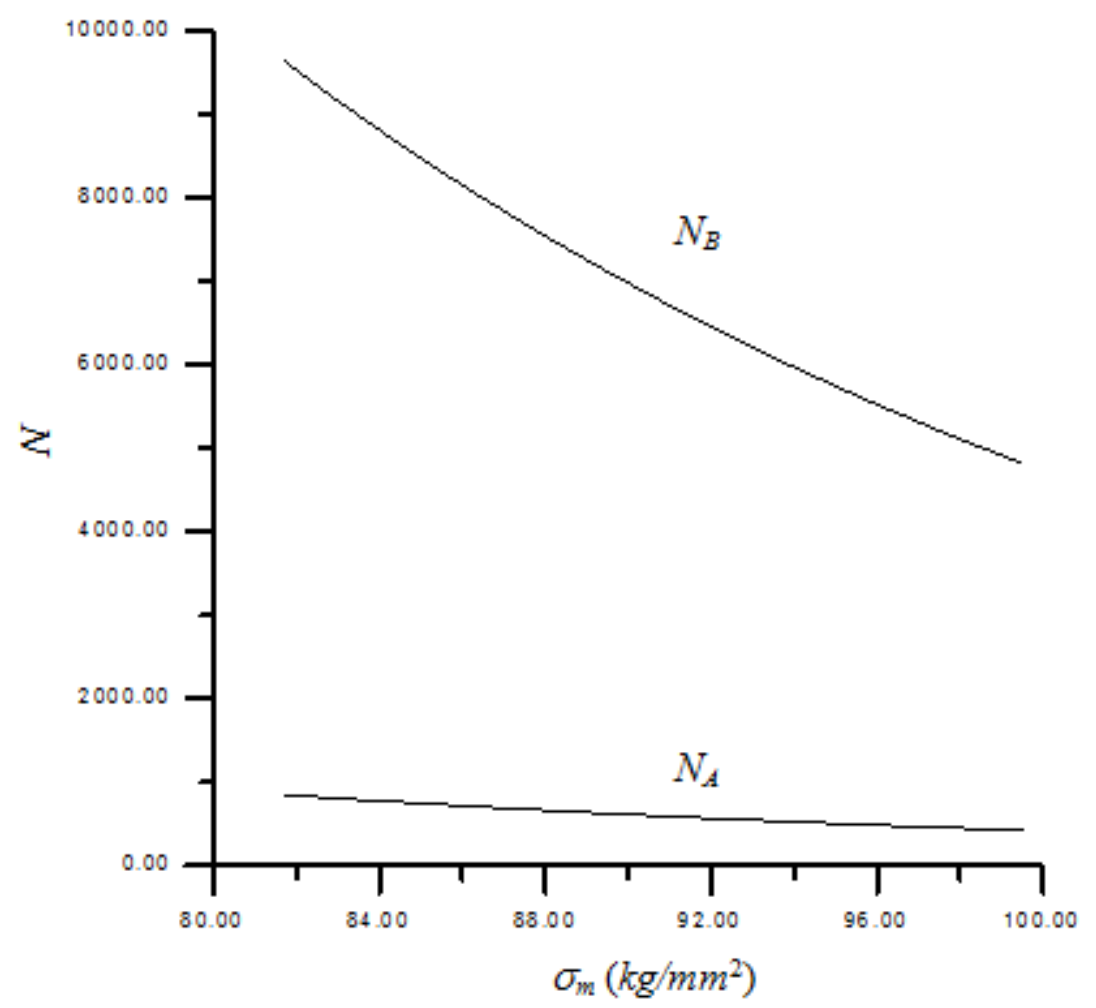

(a) U-shape bellow 


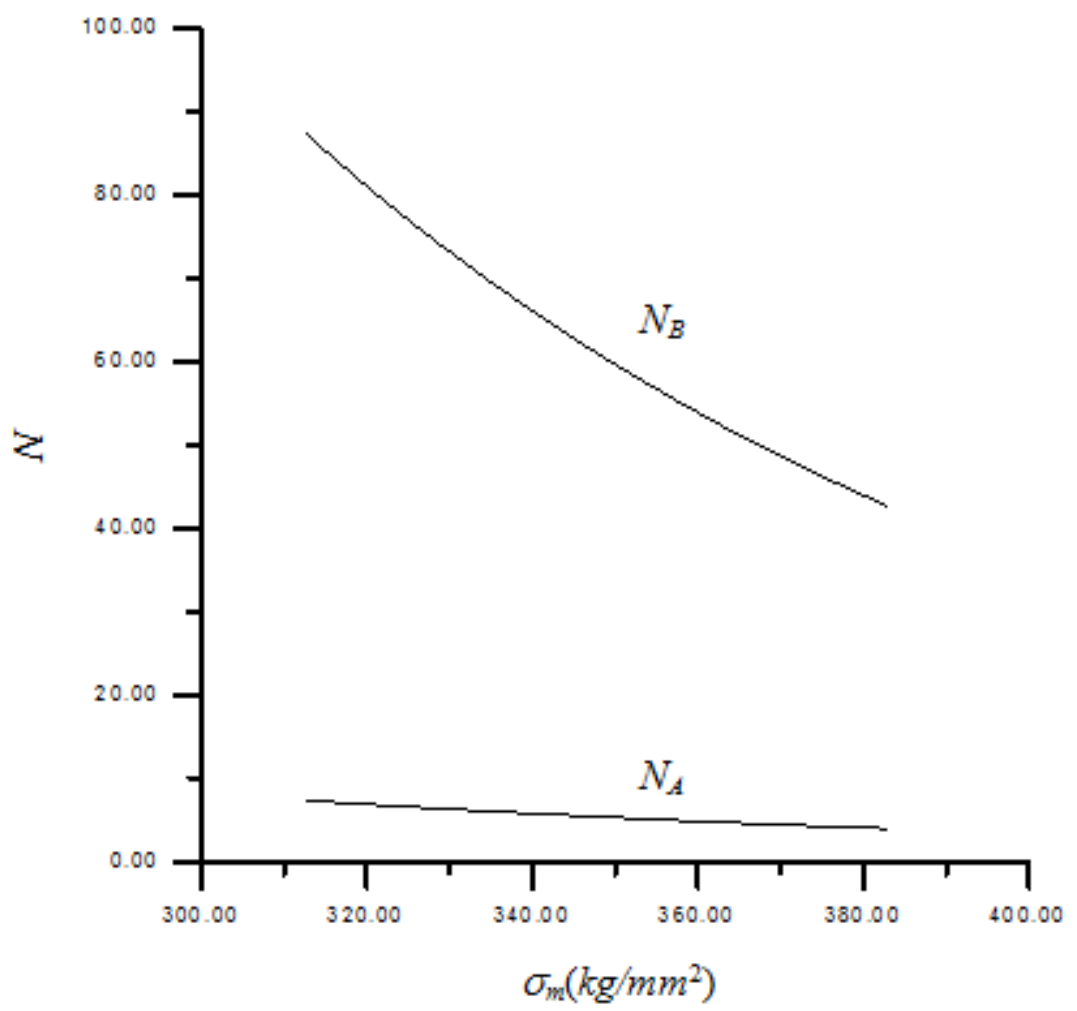

(b) $\Omega$-shape bellow

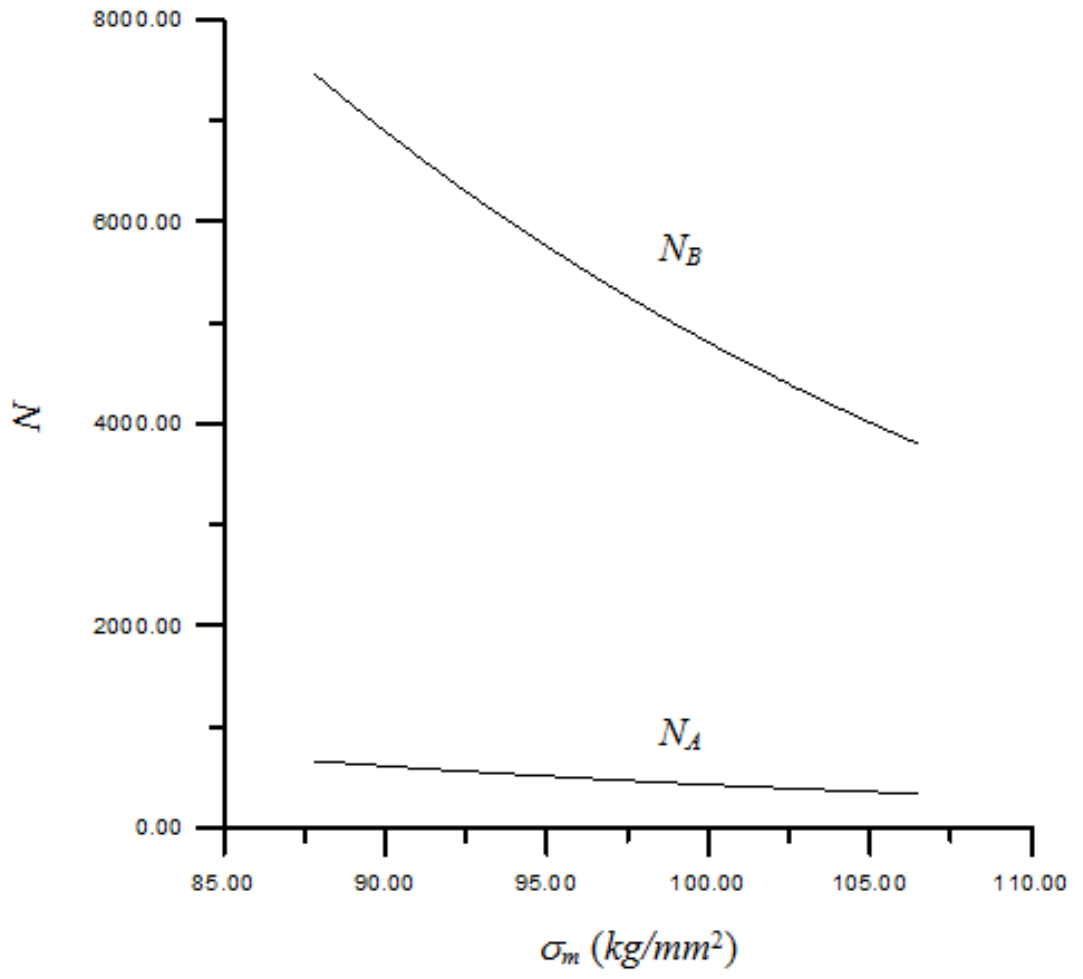

(c) disc-shape bellow

Figure 3. The relationship between ( $\sigma_{m}$ and $N$ ) for axial type with control ring, (STS 304)

For the bellow with (STS 316) material, Fig. (4) shows that the $N_{A}$ and $N_{B}$ reach to the maximum value of about $50 \times 10^{3}$ and $500 \times 10^{3}$ respectively in case of U-shape corrugation with axial type when the total stress changes from 30 to60 $\mathrm{kg} / \mathrm{mm}^{2}$.
In case of the axial type with control ring, the $N_{A}$ and $N_{B}$ reach to the maximum value of about $1.7 \times 10^{3}$ and $16 \times 10^{3}$ respectively in $U$-shape corrugation as shown in Fig. (5). 


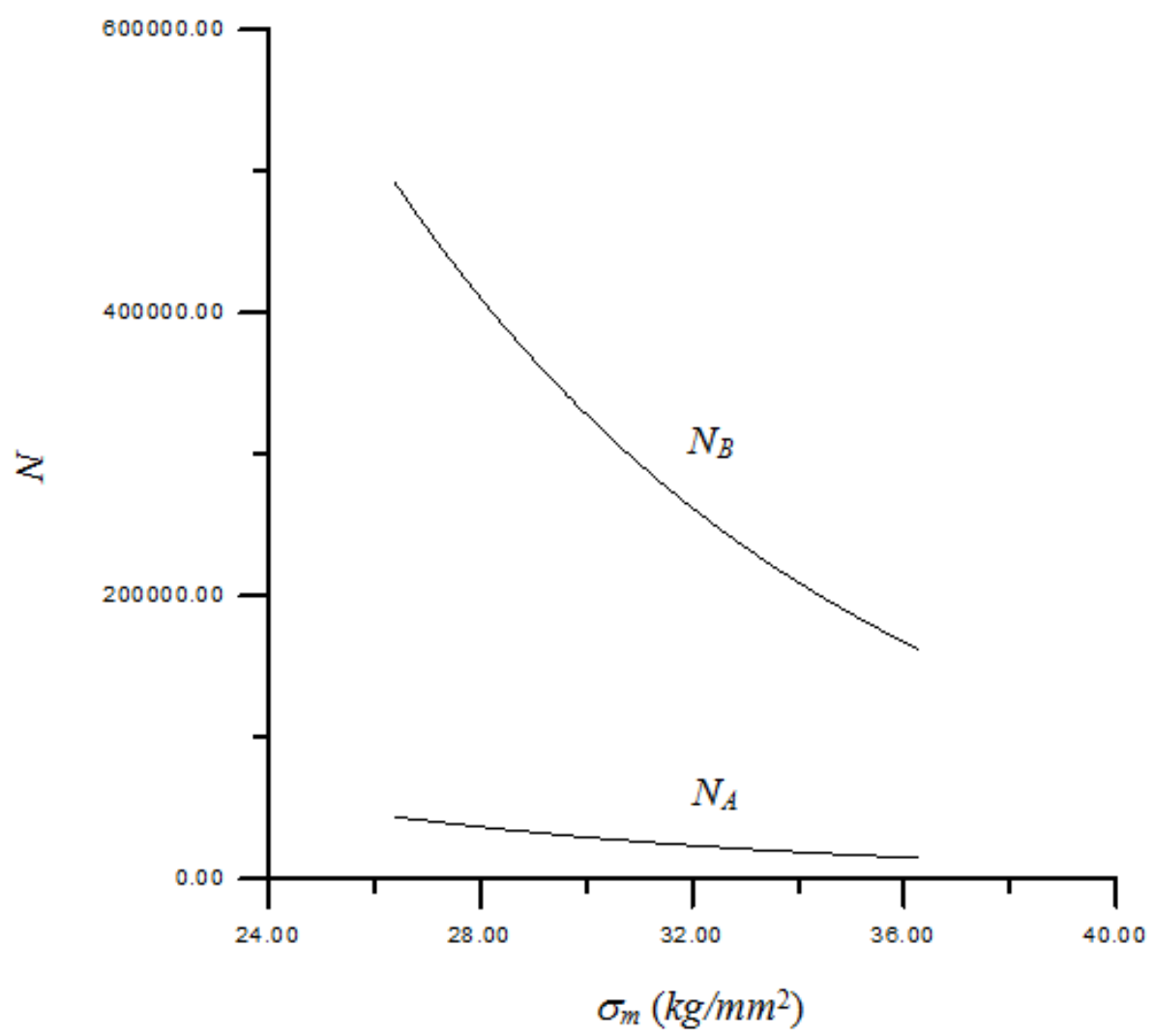

(a) U-shape bellow

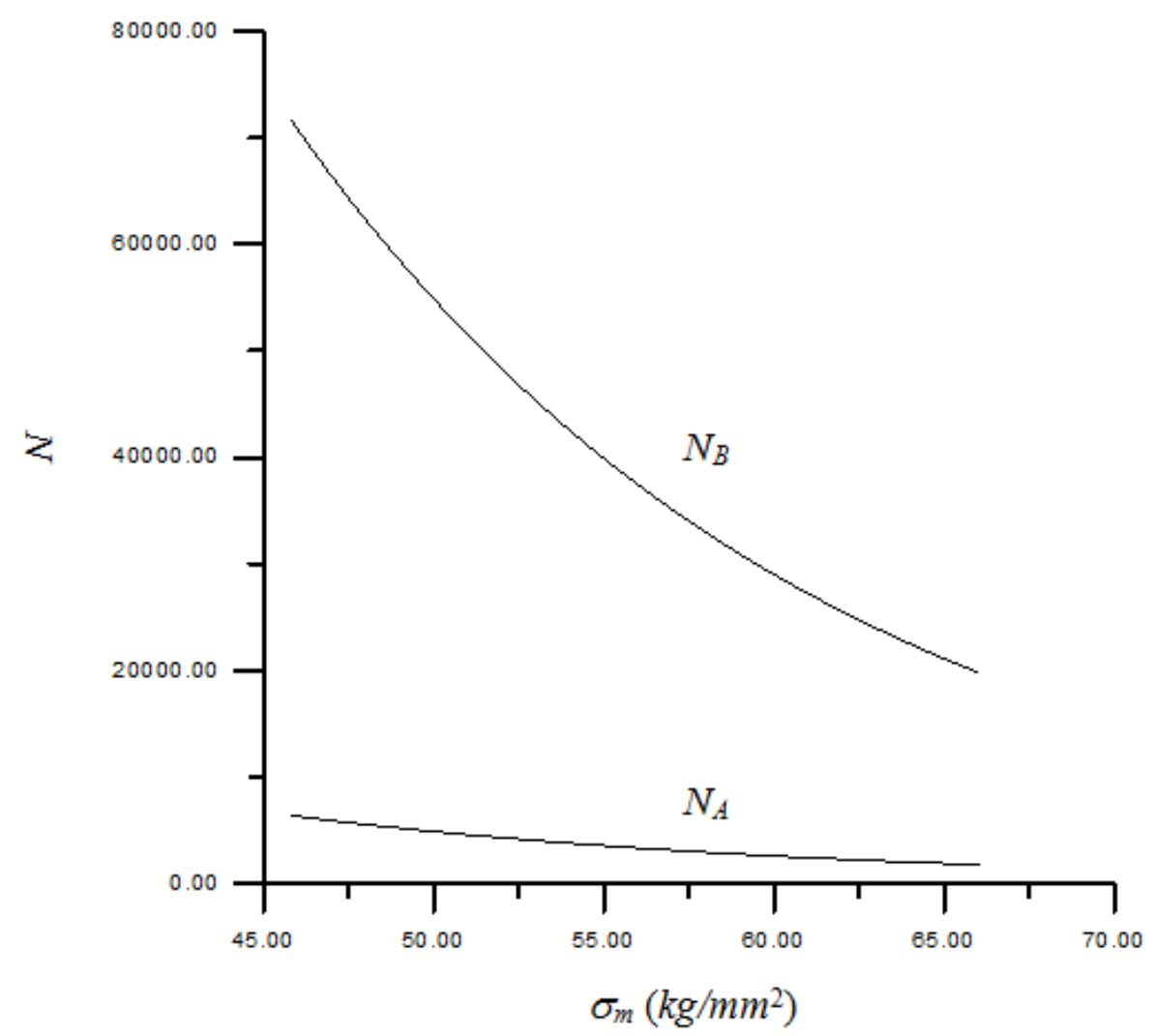

(b) $\Omega$-shape bellow 


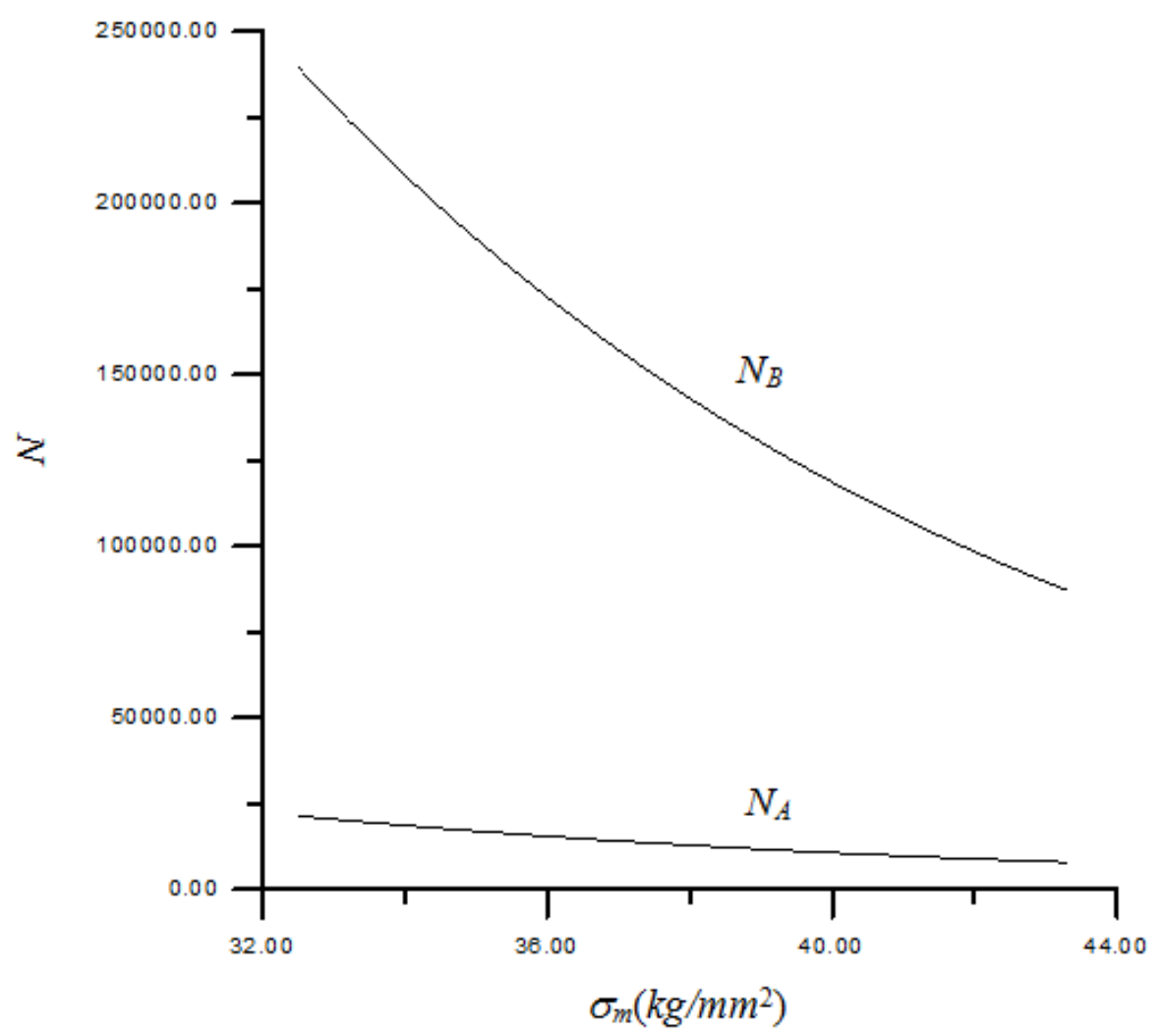

(c) disc-shape bellow

Figure 4. The relationship between ( $\sigma_{m}$ and $N$ ) for axial type, (STS 316)

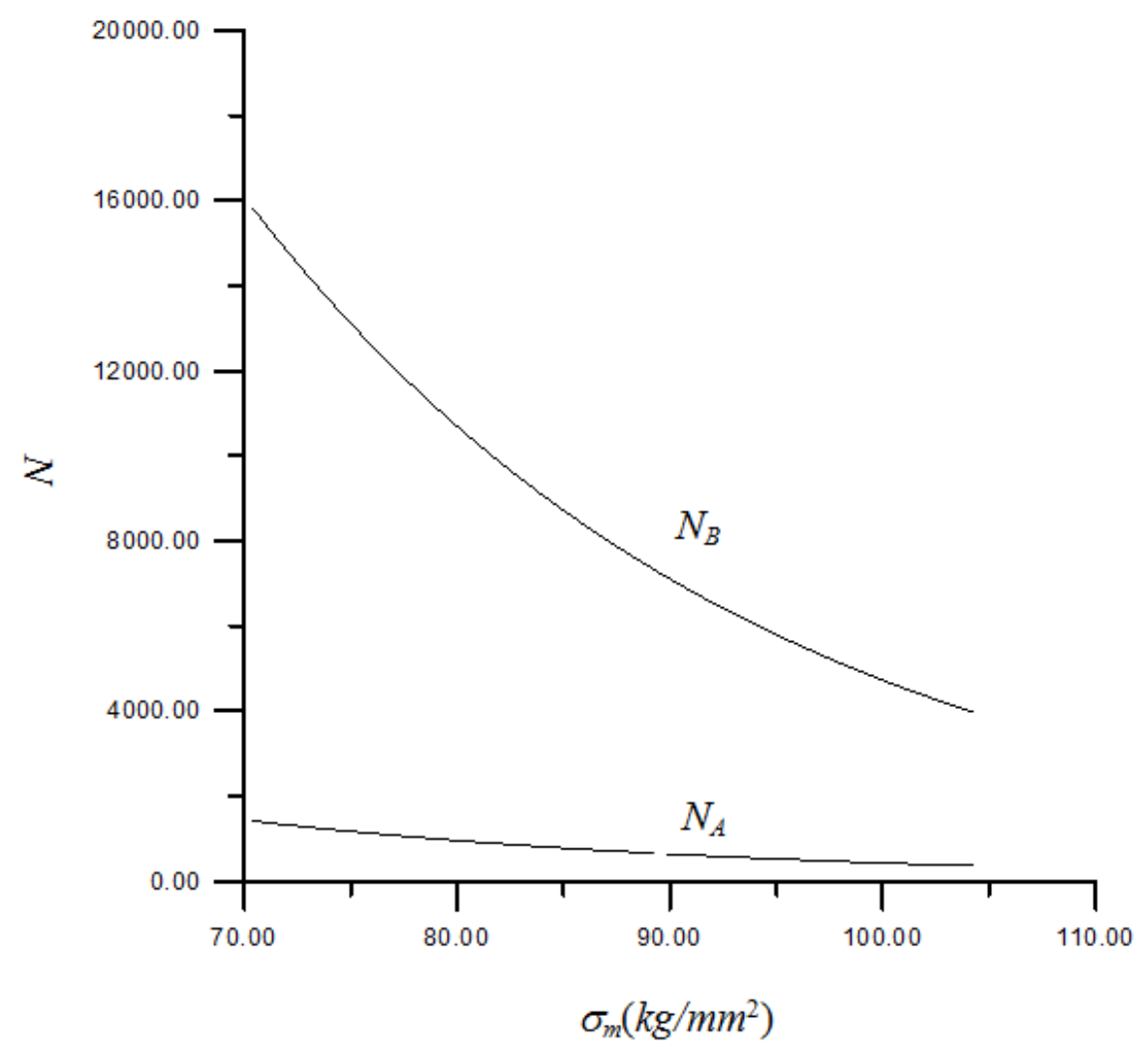

(a) U-shape bellow 




(b) $\Omega$-shape bellow

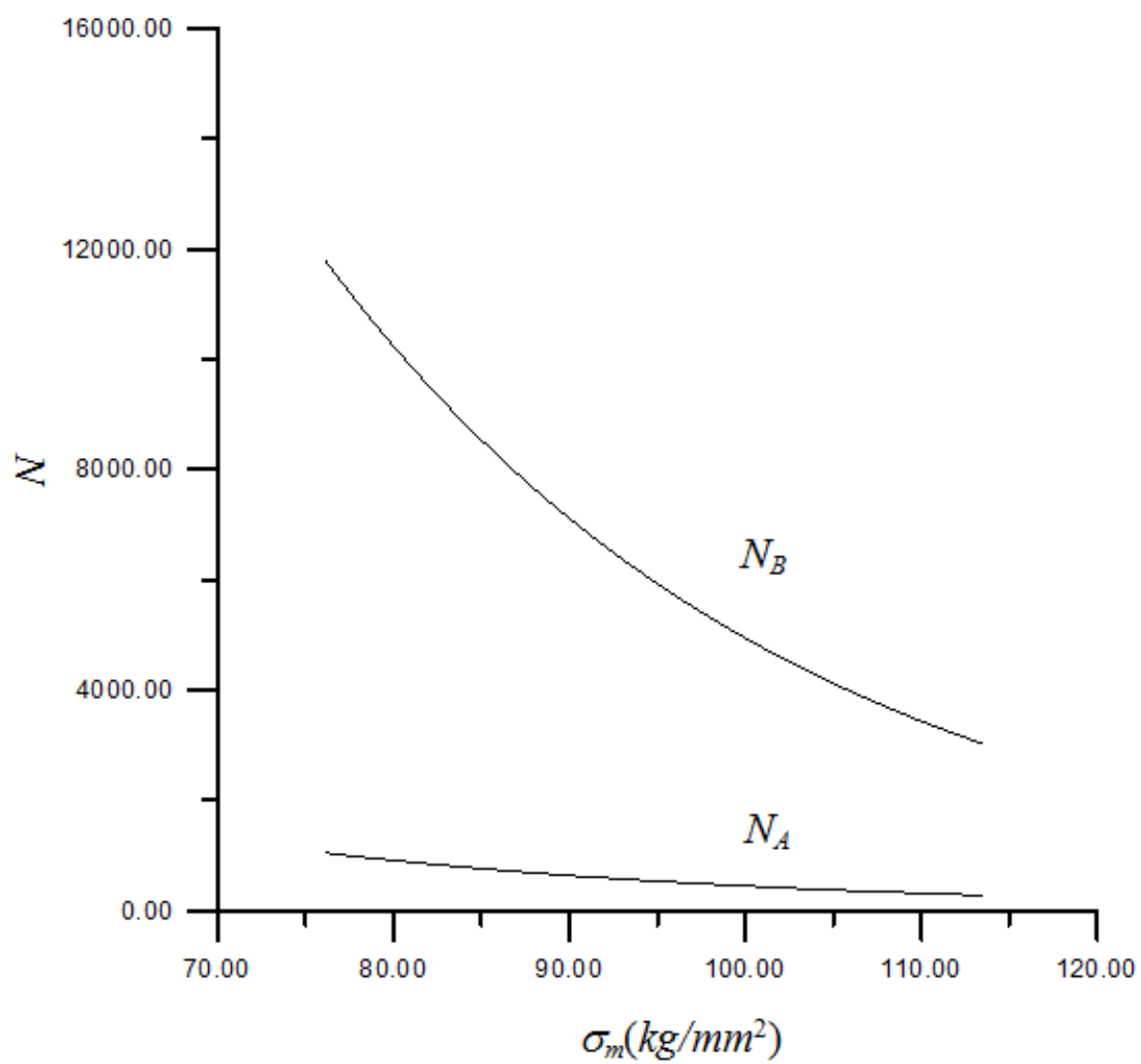

(c) disc-shape bellow

Figure 5. The relationship between ( $\sigma_{m}$ and $N$ ) for axial type with control ring, (STS 316) 


\section{Conclusions}

The relationship between maximum stresses produced and the predicted cycle life of bellows which are made of (STS) material has been presented. Simulation model is written by MATLAB based on specific design parameters of bellows. The solution procedure involves three shapes of bellow section (U-shape, $\Omega$-shape and disc-shape).

The following conclusions can be summarized:

- It is important to predict a realistic cycle life as a design consideration when ordering an expansion joint.

- Both the $N_{A}$ and $N_{B}$ are increased with decreasing of the maximum stress $\left(\sigma_{m}\right)$.

- In the case of bellows of (STS 304) material, the $N_{A}$ and $N_{B}$ reach to the maximum value of about $90 \times 10^{3}$ and $800 \times 10^{3}$ in case of U-shape corrugation with axial type, while in case of axial type with control ring, reach to the maximum value of about $1 \times 10^{3}$ and $10 \times 10^{3}$ in U-shape corrugation.

- In the case of bellows of (STS 316), the $N_{A}$ and $N_{B}$ reach to the maximum value of about $50 \times 10^{3}$ and $500 \times 10^{3}$ in case of U-shape corrugation with axial type and reach to the maximum value of about $1.7 \times 10^{3}$ and $16 \times 10^{3}$ in U-shape corrugation with axial type with control ring.

- U-shaped bellows have smaller internal pressure-induced stress, longer fatigue life, and is more suited for higher internal pressure situations.

- The correct specification of bellows movement requirements can be considered as one of the most essential factors in the design.

\section{Nomenclature}

$D_{P} \quad$ Effective diameter of bellow (mm)

$e^{\text {Axial movement per one bellow corrugation }(\mathrm{mm})}$

E Young's modulus of elasticity $\left(\mathrm{kg} / \mathrm{mm}^{2}\right)$

$H \quad$ Height of bellow corrugation (mm)

$m \quad$ Number of plies

$N_{A} \quad$ Allowable cycle life

$N_{B} \quad$ Broken cycle life

$P \quad$ Internal pressure $\left(\mathrm{kg} / \mathrm{mm}^{2}\right)$

$t \quad$ Thickness of bellow $(\mathrm{mm})$

$T \quad$ Temperature $\left({ }^{\circ} \mathrm{C}\right)$

$W \quad$ Half pitch of bellow corrugation (mm)

Greek symbols

$\sigma_{a} \quad$ Allowable tensile stress $\left(\mathrm{kg} / \mathrm{mm}^{2}\right)$

$\sigma_{e} \quad$ Stress due to deflection $\left(\mathrm{kg} / \mathrm{mm}^{2}\right)$

$\sigma_{m} \quad$ Total stress $\left(\mathrm{kg} / \mathrm{mm}^{2}\right)$

$\sigma_{P} \quad$ Stress due to internal pressure $\left(\mathrm{kg} / \mathrm{mm}^{2}\right)$

$\sigma_{y} \quad$ Yield stress $\left(\mathrm{kg} / \mathrm{mm}^{2}\right)$
Abbreviates

EJMA Expansion Joint Manufacturing Associate

\section{References}

[1] Igi, S., Katayama, H., and Kawahara, M., "Evaluation of mechanical behavior of new type bellows with two-directional convolutions," Nuclear Engineering and Design, vol. 197(1), pp. 107-114, 2000.

[2] Hyspan Technical Notes, "Bellows Movements", http://www.hyspan.com/pdfs/BellowsMovemStTechNote.pdf, 2005.

[3] http://www.flexpertbellows.com/products/technical-informatio n/cyclic-or-fatigue-life-of-metal-bellows-expansion-joints/

[4] EJMA, "The Expansion Joint Manufacturing Association Standard", http://www.ejma.org/, $8^{\text {th }}$ Edition, 2003.

[5] Li, Y., and Sheng, S., "Strength analysis and structural optimization of U-shaped bellows," International Journal of Pressure Vessels and Piping, vol. 42(1), pp.33-46, 1990.

[6] Subramanian, G., and Raghunandan, C., "On improving the fatigue life of U-form bellows," Journal of Materials Processing Technology, vol. 41(1), pp. 105-114, 1994.

[7] Becht IV, C., "Fatigue of bellows, a new design approach," International Journal of Pressure Vessels and Piping, vol.77 (13), pp. 843-850, 2000.

[8] Tingxin, L., Xiaoping, L., Tianxiang, L., Xigang, H., and Xinfeng, L., "Experimental research of toroid-shaped bellows behavior," International Journal of Pressure Vessels and Piping, vol. 63(2), pp. 141-146, 1995.

[9] Zhu, Y. Z., Wang, H. F., \& Sang, Z. F., "The effect of environmental medium on fatigue life for u-shaped bellows expansion joints," International Journal of Fatigue, vol. 28(1), pp. 28-32, 2006.

[10] Pierce, S. O., \& Evans, J. L., "Failure analysis of a metal bellows flexible hose subjected to multiple pressure cycles," Engineering Failure Analysis, vol. 22, pp. 11-20, 2012.

[11] http://www.expansionjointsindia.com/Questionnaire.pdf

[12] Hammond M. G., "Quite Hydraulic Actuation Bellows Design Considerations", www: http://www.ligo.caltech.edu, 2002.

[13] Expansion Joint \& Flexible Product, Fabrication procedure, http://www.megaflexon.com/design/default/images/Introducti on_Metallic\%20Expansion\%20Joint.pdf

[14] Edward B.M., Shapour A., Balakumar B., James D. Keith H. Gregory W., An engineer's guide to MATLAB : with applications from mechanical, aerospace, electrical, and civil engineering, Prentice Hall (C2011, 3rd edition, 2011. 